\title{
Copyright law and multimedia- or Internet-based educational applications
}

\author{
DAVID A. WASHBURN \\ Georgia State University, Atlanta, Georgia \\ and Center of Excellence for Research on Training, Atlanta, Georgia
}

\begin{abstract}
Advances in computer technology, in combination with the resources of the Internet, afford the educator an unprecedented array of options for facilitating instruction. At the same time, this technology introduces numerous ways of inadvertently or intentionally violating federal copyright law and guidelines. Copyright law, the principles of fair use, and guidelines for reproduction of text and off-air broadcast media will be discussed, with specific reference to the development of multimedia programs and Internet-based applications.
\end{abstract}

Recent years have witnessed amazing developments in computer technology, both in terms of the capabilities of available hardware and software and also with respect to its affordability. As a consequence, the desktop computer that became a common tool for educators ${ }^{1}$ just a few years ago is now connected to color printers, text and graphic scanners, video capture boards, digital cameras, and a compact disc and sound system that rivals most entertainment units. In addition, the modem that resides in that computer permits connection to the Internet and the wealth of text, images, sounds, and information on the World-Wide Web (WWW). For a few dollars more, the average educator can also acquire software for producing professional-looking graphics, multimedia presentations, and WWW sites.

There has been substantial discussion already regarding opportunities and options for applying these resources to the goals of education and training. At the 1996 meeting of the Society for Computers in Psychology alone, there were three symposia related to this topic. Unquestionably, this trend will continue at many conferences and in journals such as Teaching of Psychology and the present periodical. Potential applications that have been described include using computers to generate better transparencies and handouts, developing computerized drills and simple computer-based education programs, employing panel projectors to display computer-generated visual aids, integrating lecture and visual information into multimedia packages, using electronic mail and list pro-

This paper was supported by Contract DAAH04-96-BAA6 from the Army Research Office to the Center of Excellence for Research on Training and by Grant NAG2-438 to Georgia State University. The author thanks John Marshall, Jr., for his assistance. For reprints and further information, address correspondence to D. A. Washburn, Department of Psychology, Georgia State University, Atlanta, GA 30303 (e-mail: dwashburn@gsu.edu). cessors to distribute materials and to facilitate discussion, posting lecture notes or other information on the WWW, and offering on-line courses or other distance learning options.

With this array of power and promise, however, come several serious challenges. At issue for the present discussion is the increasing risk that educators who try to develop computerized instructional materials might inadvertently or intentionally violate copyright law and the principles of fair use. Not only does contemporary technology make it easier to violate copyright law, but the WWW can amplify the seriousness of these violations, as well as the likelihood of getting caught. As an example, consider the following hypothetical scenario.

A professor wishes to develop a computerized instructional tool for use in a history of psychology course she teaches. To replace the lengthy passages of sundry classic books that she typically reads aloud to the students, she transcribes the readings into a compilation and downloads an accompanying photo of each historical figure from the WWW. She also digitizes segments from a documentary on Freud that she had taped from public television a few years earlier. In the past, the students have reviewed the tape positively, and the digitized version will eliminate the need to scan and rewind to the relevant sections of the program. Several seminal papers are not in the library, so, to encourage students to read these original sources, she scans and posts each publication (with appropriate citation information) on her departmental Web page, along with study guides provided by the textbook publisher. These applications work so successfully that the professor gives a demonstration of the multimedia and WWW tools at a professional conference. Several colleagues at the conference ask for a copy of her paper, so she makes it available to them on her Web page and submits it for publication. Although the computerized instructional package requires substantial time and 
effort to develop, she is encouraged by the response from students and colleagues and by the time that she will save in future terms by using the tool.

It seems safe to assert that such an instructional package would be the envy of educators everywhere. Unfortunately, it is also likely that the hypothetical professor in this scenario violates copyright law-perhaps at every stage of development! To show why this might be the case, I will briefly outline copyright law and the doctrine of fair use, guidelines that are relevant to educational multimedia and WWW applications, and common misconceptions about copyright issues. ${ }^{2}$ Subsequently, I will discuss the copyright consequences of developing original multimedia and Internet-based educational works (i.e., those that do not contain material copyrighted by another owner). Finally, WWW resources for further information on these issues will be discussed.

\section{OVERVIEW OF COPYRIGHT ACT AND FAIR USE}

Contrary to common perception, copyright law is designed primarily to promote creativity, education, scholarship, and the expression of culture and only secondarily to protect or reward the copyright owner (Kasunic, 1992). This is not to suggest that copyright owners are without rights or legal recourse in the case of infringement. In fact, violating a copyright owner's exclusive rights of duplication, modification, distribution, performance, or display can result in injunction and liability for damages of up to $\$ 100,000$. However, when the Copyright Act of 1909 was revised in 1976, Congress codified the doctrine of fair use (long a common law principle in copyright law, but statutory beginning in 1978) in order to limit these exclusive rights and to provide for the reproduction of copyrighted material "for purposes such as criticism, comment, news reporting, teaching (including multiple copies for classroom use), scholarship, or research" (Copyright Act of 1976, section 107).

Not all educational use of copyrighted material is fair use, however. The fair use defense is based on four factors or considerations: (1) the purpose and character of the use, including whether such use is of a commercial nature or is for nonprofit educational purposes; (2) the nature of the copyrighted work; (3) the amount and substantiality of the portion used in relation to the copyrighted work as a whole; and (4) the effect of the use on the potential market for or value of the copyrighted work. Of course, these factors leave many ambiguities in the interpretation of copyright law. There are no legal definitions of when a work becomes fixed and copyrighted, of how much can be used as fair use, of the distinction between expression (which is protected by copyright) and idea (which is not), and of what constitutes substantiality or the essence of the work. These issues have been and will continue to be clarified in case law.
Recognizing these ambiguities, Congress commissioned a panel of educators, authors, and publishers to negotiate an agreement regarding permissible educational uses of copyrighted material. In 1976, the "Agreement on Guidelines for Classroom Copying in Not-For-Profit Educational Institutions With Respect to Books and Periodicals" (hereafter called the Guidelines) was produced. The Guidelines (see Appendix A) were designed to specify the minimum standards of fair use, so that educators had clear understanding of what is not a violation of copyright. This does not mean, however, that violations of the Guidelines are necessarily violations of fair use and copyright law, as the Guidelines were never intended to state the maxima or limits of fair use (see Kasunic, 1992, for additional discussion).

In the years since introduction of the Guidelines, however, they have been seen and used as the maximum standards that they were never intended to be. Publishers have promoted the Guidelines as the definition of fair use, and many universities have adopted them as policy. Similar guidelines have also been published for the use of music, off-air videotaping, and software (including multimedia)- each with similarly restrictive results. Although these guidelines (see Appendix A) are not law, they do define a safe zone that university administrators respect and beyond which the educator risks an interpretation from the courts.

The latest body of guidelines-particularly relevant for the present discussion-was compiled by the Information Infrastructure Task Force (IITF) in 1995. Their presidential white paper, the IITF Report, reviewed the question of whether current copyright law is adequate to cover issues of cyberspace. Members of the IITF concluded that the Copyright Act and the principles of fair use required only minor updating, not major overhaul, to incorporate advances in technology into current copyright law. Although this conclusion has generated substantial debate, the immediate implication of the IITF Report is that the Copyright Act of 1976 with its principles of fair use-and the Guddelines that clarify these principles-will continue to dictate what constitutes fair use and what constitutes copyright infringement with respect to educational multimedia and applications on the Internet.

Many have argued that the IITF Report amplifies the maximalist error begun with the Guidelines. In an open letter to the vice president and numerous members of Congress, for example, more than 100 law professors objected to the IITF Report and to the restrictive interpretation of fair use (this letter, response, and rejoinder were posted to the WWW by Boyle, 1997). According to this letter, as well as to similar arguments published or posted elsewhere, the recommendations of the IITF Report do indeed reflect radical measures rather than the minor adjustments claimed by the IITF. Of course, the IITF Report, like the Guidelines, are not law. In addition to rep- 
resenting presidential policy, however, the arguments in the IITF Report have been brought before Congress for congressional action. To date, several bills based on these recommendations have been presented to both houses of Congress without passage.

\section{COPYRIGHT AND FAIR USE QUIZ}

In summary, the Copyright Act was written to provide educators the freedom to use published and otherwise copyrighted material in creative instructional or scholarly ways. The various guidelines and agreements that interpret this law, however, suggest that, without prior permission from the copyright owners, it is virtually impossible defensibly to incorporate copyrighted text, video, sounds, or images into instructional multimedia programs or WWW pages. Thus, the current understanding - and misunderstandings - of copyright law and the doctrine of fair use as it pertains to educational multimedia or Internet-based applications can be summarized by the following questions and answers (see also Brinson \& Radcliff, 1996; Templeton, 1997; and Appendix B, this paper).

As long as my activities are educational, am I protected by the principle of fair use? No. The purpose of the use (educational vs. for-profit, for example) is only one factor that determines fair use. As was discussed above, the nature and substantiality of the work and any impact on value must also be considered.

Must copyrighted materials specify that they are copyrighted? No. Works created after April 1, 1989, are legally copyrighted, whether they contain a copyright notice or not. Unintentional violation does not reduce liability.

Can electronic works be copyrighted? Yes. Intellectual property is copyrighted when it becomes fixed, which occurs whenever it can be perceived, reproduced, or communicated. Works are fixed when they are written on paper, spoken onto audio tape, or entered into computer memory. There is some public domain information on the WWW that is not copyrighted. However, it is likely that many of the text files, images, and sounds that are posted on the Internet already represent copyright infringements. You are liable if you copy this information (and there is substantial debate as to whether merely viewing the information, which requires loading it into computer memory, represents copying). In addition, it appears that your Internet service provider (e.g., your university) can also be held liable for your copyright law violations (Price, 1996).

Do I avoid copyright violation if I give authorship credit? No. Acknowledging the source provides a defense against charges of plagiarism but not against copyright infringement.

Can I use the work if I obtain permission from the copyright owner? Yes. Obtaining permission is the best way to use copyrighted works without liability. However, it is sometimes difficult to determine who owns a copyright or how to contact them, and of course the owner may deny use of his/her intellectual property without compensation.

Can I use copyrighted works so long as I do not profit from their use? Can I use copyrighted works so long as the owner does not lose profit from my use? If I am providing free advertising for the copyrighted material and the owner will probably profit from my use, do I avoid liability? No, No, and No. The character of use and the effect on the market are only two factors that have weight in fair use. You may still be liable. The copyright owner (and ultimately the courts) will decide what free advertising, if any, is desirable and legal.

Can I use copyrighted material once the copyright has expired? Yes. However, the fact that a work is old does not mean that it is public domain or without market value. The term of a copyright depends on who created the work and on when and why it was created and distributed. For works copyrighted after January 1, 1978, owners retain exclusive rights for the life of the owner plus 50 years-unless the owner gives the copyright away. Works for hire are generally protected for 75 years beyond the date of first publication or distribution.

Even if I violate copyright law, isn't it unlikely that my infringement will be detected or prosecuted? No. Copyright infringements at the photocopy machine may be difficult to detect, but the Internet affords unparalleled connectivity between individuals - across disciplinary and geographic boundaries. It is increasingly easy for copyright owners to search for potential violators. Furthermore, given the number of people that gain access to works when posted on the WWW, it may increasingly be financially worthwhile for owners to prosecute infringements.

\section{COPYRIGHT ISSUES FOR ORIGINAL WORKS}

One can, of course, avoid the risk of copyright infringement by constructing multimedia or Internet-based instructional tools that contain only original works. Note that one cannot merely alter previously copyrighted text or images to produce new works free of copyright protection, as these derivative works are also protected by copyright. On the other hand, an educator possesses exclusive rights to the reproduction, modification, distribution, performance, and display of original works, such as manuscripts, graphs or art, sounds, or images. These may be packaged into multimedia programs or posted on the Internet.

Of particular note for the present discussion, however, are the consequences of posting a manuscript for distribution, comment, or review on the WWW. As author and copyright owner, one can legally post unpublished manuscripts and can seek punitive damages against individuals who infringe on the owner's exclusive rights. However, it is generally inadvisable to do so. After contacting several major journal publishers in psychology, I learned that the posting of unpublished manuscripts can jeopardize publication. The policy for journals published by the American Psychological Association, for example, is currently available at www.apa.org/journals/posting.html

As Burnette (1996) notes, authors are advised not to put manuscripts on the Internet at any stage (draft through publication). Although the copyright can be transferred from author to publisher in any case, the American Psy- 
chological Association views manuscripts posted on the Internet as previously published and, as such, will not consider them for their journals. In response to my inquiry, Psychonomic Society Publications indicated that their policy was similar. The posting of manuscripts, in whole or major part, on the Internet will jeopardize journal publication. Of the five journal publishers contacted, only Academic Press expressed a different policy. Academic Press currently accepts submissions that are posted on the Internet, so long as the postings inform readers that the manuscript was "accepted for publication" by one of their journals. All publishers contacted believed that the posting of papers after they had been published represented a violation of the publisher's copyright. None of the publishing companies objected to the posting of abstracts on the Internet, either before or after journal publication.

\section{DISCUSSION}

At the beginning of this paper, I discussed a fictitious psychology professor and her efforts to integrate instructional technology into a history and systems course. According to the guidelines and policies discussed here, this professor has risked copyright violation in numerous ways. Producing a compilation from printed matter (e.g., classic books or seminal articles) and reproducing published readings into digital form would appear to be clear infringements. With respect to off-air recordings, not only would it be a violation to digitize segments of a program videotaped from public broadcasting, but the guidelines would further suggest that the mere classroom use of the videotape on a regular basis is a violation of the copyright owner's rights. In addition, it is clear that posting a manuscript on the WWW currently jeopardizes any subsequent professional publication. Thus, it seems likely that copyright owners would have strong claims against our well-intentioned teacher, who has left the safe harbor outlined by the guidelines in her pursuit of improved instruction.

How would the courts decide in the case against our hypothetical professor? In violating the various guidelines, has she indeed broken the law and the doctrine of fair use? This is uncertain. Educators, publishers, lawmakers, and lawyers are still scrambling to understand the impact of copyright law on educational technology and the impact of technological changes on copyright law. For example, the policies just discussed regarding electronic publication of manuscripts are interim and subject to ongoing investigation and modification. Although the Copyright Act, the principles of fair use, and the subsequent guidelines are relatively fixed, the clarification of these laws and interpretations is ongoing. It is important for educators to understand these limits and standards. It is also essential for educators to lobby Congress and university administrations in defense of fair use. The technologies that are currently available could make dramatic improvements in the quality and efficiency of education. Realizing these benefits in the classroom, however, will require both the creativity of educators and also their willingness to defend and ensure that copyright law and policy reflect the best interests of education.

\section{REFERENCES}

BOYLE, J. (1997). Open letter regarding white paper. Unpublished document available on Internet: http://www.clark.net/pub/rothman/ boyle.htm

BRINSON, J. D., \& RADCLIFF, M. F. (1996). An intellectual property law primer for multimedia and web developers. Unpublished document available on Internet: http://www.eff.org/pub/CAF/law/ip-primer

Burnette, E. (1996). Publishing papers on the Internet could pose problems. APA Monitor, $27,15$.

COPYRIGHT ACT OF 1976, I7 U.S.C. \$§ IOI-9I4 (1988)

Kasunic, R. (1992). Fair use and the educator's right to photocopy copyrighted material for classroom use. Journal of College \& University Law, 19, 271-293.

Price, J. R. (1996). Colleges and universities as Internet service providers: Determining and limiting liability for copyright infringement. Journal of College \& University Law, 23, 183-229.

TEMPI.ETON, B. (1997). Ten big myths about copyright explained. Unpublished document availabie on the Internet: http://www.clari.net/ $\mathrm{brad} /$ copymyths.html

\section{NOTES}

1. The present discussion will focus on educators and educational applications. Clearly, these same issues are important for human factors and industrial/organizational psychologists, such as those who study training in industry and the military. Indeed, individuals developing computerized training tools must be even more cautious, as they frequently lack the defense of fair use.

2. This discussion is provided for information only. Readers should consult the Copyright Act of 1976, the relevant guidelines, and the policies of their institution before using works governed by copyright law.

\section{APPENDIX A}

\section{Copyright Compliance Guidelines}

The following pages summarize agreements on guidelines for copyright compliance that are relevant to multimedia- or Internet-based educational applications. This information is provided to complement the present discussion only. Before developing a computerized educational application, readers are advised to check the Copyright Act of 1976, the full text of each of these guidelines, and their university policy.

A. Books and periodicals (summarized from the Agreement on Guidelines for Classroom Copying in NotFor-Profit Educational Institutions With Respect to Books and Periodicals)

1. Teachers may make a single copy for scholarly research or teaching of

a. chapter from a book

b. an article from a periodical or newspaper

c. a short story, short essay, or short poem

d. a chart, graph, diagram, drawing, cartoon, or picture from a book, periodical, or newspaper 
APPENDIX A (Continued)

2. Teachers may make multiple copies (not to exceed one copy per pupil in a course) for classroom use or discussion, provided that

a. the copying is limited to

i. 250 words of a poem

ii. an entire 2,500-word article, story, or essay

iii. no more than 1,000 words or $10 \%$ (whichever is less) of a longer prose work

iv. one chart, graph, diagram, drawing, cartoon, or picture per book or per periodical issue

v. no more than $10 \%$ of "special" works

b. the copying is at the instance and inspiration of the individual teacher

c. the inspiration and decision to use the work and the moment of its use for maximum effectiveness are so close in time that it would be unreasonable to obtain permission in time

d. the copying of the material is for only one course

e. not more than one short poem, article, story, essay, or two excerpts may be copied from the same author nor more than three from the same volume

f. there are no more than nine instances of multiple copying for one course during one class term

g. each copy includes notice of copyright

3. Prohibitions:

a. Copying shall not be used to create or substitute for anthologies, compilations, or collective works.

b. No copying of materials intended to be "consumable."

c. Copying shall not

i. substitute for the purchase of books or reprints

ii. be directed by higher authority

iii. be repeated (same item, same teacher) term-to-term

d. No charge made to students beyond cost of copying.

B. Off-air broadcast copying (summarized from Guidelines for Off-Air Recording of Broadcast Programming for Educational Purposes)

1. A broadcast program may be recorded off-air and retained by a nonprofit educational institution for a period not to exceed 45 days from the broadcast. After this period, the recording must be erased.

2. Off-air recordings may be used

a. once by individual teachers in the course of relevant teaching activities and repeated once only when instructional reinforcement is necessary

b. in classrooms or similar campus places

c. during the first 10 days of the retention period, after which the recording is retained only for evaluation by teachers

3. Off-air recordings may be made only by request of individual teachers and may not be made routinely in anticipation of requests.

4. Off-air recordings cannot be altered or combined to form anthologies or compilations.

5. All off-air recordings must include copyright notice.

C. Multimedia (summarized from Fair Use Guidelines for Educational Multimedia, prepared by the Consortium of College and University Media Centers Fair Access Working Committee, 1995)

1. Educators may use lawfully acquired copyrighted works in producing multimedia programs for their own teaching tools in support of an identified curriculum.

2. Educators may perform or display their own multimedia programs at workshops or conferences of their peers.

3. Educators may use lawfully acquired copyrighted materials in their own multimedia programs over the institution's electronic network, provided that there are technological limitations on access (e.g., password or PIN).

4. Educators may use their own multimedia programs in teaching courses for up to 2 years. Use beyond this time requires permission for copyrighted works.

5. Multimedia programs must comply with these portion limits

a. Motion media: up to $10 \%$ or $3 \mathrm{~min}$, whichever is less

b. Text material: up to $10 \%$ or 1,000 words, whichever is less

c. Music: up to $10 \%$ or $30 \mathrm{sec}$, whichever is less

d. Illustrations and photographs: no more than 5 images of an artist or photographer; no more than $10 \%$ or 15 images from a collective work

6. Permission must be obtained for copyrighted works to be included in multimedia programs
a. for commercial distribution
b. before making more than one copy
c. that represent interinstitutional collaboration
d. that will be used over uncontrolled electronic networks 


\section{APPENDIX B \\ Internet Resources for Copyright Law Information}

The WWW has a wealth of information on domestic and international copyright law, guidelines and agreements, fair use, and frequently asked questions. Below are some of the sites that were useful in the preparation of the present report and that contain links to networks of additional information.

URL: http://www.iupui.edu/it/copyinfo/fairuse.html

Contact: Copyright Management Center, Indiana University-Purdue University, Indianapolis

Title: Copyright and Fair Use: Their Meaning for Higher Education

Contents: links to relevant information, selected readings

URL: http://arl.cni.org/info/frn/copy/copytoc.html

Contact: Association of Research Libraries

Title: Copyright and Intellectual Property

Contents: links and information, particularly for multimedia

URL: http://www.law.cornell.edu/topics/copyright.html

Contact: Cornell University School of Law

Title: Copyright Law Materials

Contents: links to Copyright Act of 1976, case law, references

URL: http://www.ciesin.ee/other/copyinfo.html

Contact: Todd Haugland, Virginia Tech

Title: Guide to U.S. Copyright Law as Applied to Multimedia Productions

Contents: sample request and release forms

URL: http://www.clark.net/pub/rothman/boyle.htm

Contact: David H. Rothman

Title: 100+ Law Professors Criticize White House's Radical Paper on Intellectual Property

Contents: Boyle's "open letter" and debate on the IITF Report

URL: http://www.clari.net/brad/copymyths.html

Contact: Brad Templeton

Title: 10 Big Myths About Copyright Explained

Contents: common misconceptions

URL: http://www.eff.org/pub/CAF/law/ip-primer

Contact: Nancy Brinson or Mark Radcliff

Title: A Copyright Law Primer for Multimedia and Web Developers

Contents: Copyright protection for multimedia and Web developers.

(Manuscript received October 15, 1997;

revision accepted for publication January 6,1998 .) 